SLAC-PUB-8442

April 2000

UCSD/PTH-00-10

\title{
The Superpartner Spectrum of Gaugino Mediation
}

\author{
Martin Schmaltz ${ }^{x}$ and Witold Skiba ${ }^{y}$ \\ ${ }^{x}$ Stanford Linear Accelerator Center \\ Stanford University, Stanford, CA 94309 \\ ${ }^{\mathrm{y}}$ Department of Physics, \\ University of California at San Diego, La Jolla, CA 92093
}

Submitted to Physical Review D

Stanford Linear Accelerator Center, Stanford University, Stanford, CA 94309

Work supported by Department of Energy contract DE-AC03-76SF00515. 
SLAC-PUB-8442

UCSD/PTH-00-10

hep-ph/yymmnnn

\title{
The Superpartner Spectrum of Gaugino Mediation
}

\author{
Martin Schmaltz ${ }^{x}$ and Witold Skiba ${ }^{y}$ \\ ${ }^{x}$ SLAC, Stanford University, Stanford, CA 94309 \\ schmaltz@slac.stanford.edu \\ ${ }^{y}$ Department of Physics, University of California at San Diego, La Jolla, CA 92093 \\ skiba@einstein.ucsd.edu
}

\begin{abstract}
We compute the superpartner masses in a class of models with gaugino mediation (or no-scale) boundary conditions at a scale between the GUT and Planck scales. These models are compelling because they are simple, solve the supersymmetric flavor and CP problems, satisfy all constraints from colliders and cosmology, and predict the superpartner masses in terms of very few parameters. Our analysis includes the renormalization group evolution of the soft-breaking terms above the GUT scale. We show that the running above the GUT scale is largely model independent and find that a phenomenologically viable spectrum is obtained.
\end{abstract}




\section{INTRODUCTION}

The soft-breaking terms in the Minimal Supersymmetric Standard Model (MSSM) need to have a very special form for the model to be viable. Generic mass matrices for the squarks and sleptons lead to unacceptably rapid flavor-changing and lepton-number-violating processes. Similarly, the tri-linear soft-breaking breaking A-terms also require fine tuning for the MSSM to agree with experiment. It is important to explore models which naturally solve the flavor fine-tuning problem.

The rates for flavor-changing processes induced by the soft masses are proportional to the ratios of the off-diagonal mass squares to the diagonal ones. In order to make such ratios small one needs to minimize the off-diagonal terms or increase the flavor-preserving masses. Examples of natural scenarios are gauge mediation [1,2] and anomaly mediation [3], which produce diagonal mass matrices due to the universality of the gauge coupling, and effective supersymmetry [4], which postulates large soft masses for the first two generations, for which the experimental constraints are most stringent. The popular minimal supergravity [5] model does not contain a solution to the flavor problem, instead one simply assumes that the higher-dimensional operators which produce the scalar masses are flavor preserving.

It has been noticed that in "gaugino-dominated" models the flavor problem is less severe [6]. If at a high scale gaugino masses are larger than other soft parameters at that scale, then at low energies the soft masses consist of large flavor-conserving masses with smaller flavor-violating components. This is because the renormalization group running induces (positive!) universal soft scalar masses proportional to the gaugino masses. The generated scalar masses are flavor universal because couplings to gauginos are generation-independent. The most appealing gaugino-dominated scenarios have no soft masses for squarks and sleptons and no A-terms at a high scale $M_{B C}$. Since at the high scale there are no masses and no A-terms, the only sources of flavor violation are the Yukawa matrices, thus the supersymmetric flavor problem is solved by a "super-GIM" mechanism [7].

Gaugino domination also alleviates the supersymmetric CP problem because the only sources for new phases are the gaugino masses, $\mu$, and $B$. Two phases can be rotated away by field re-definitions, leaving only one possible new phase. For the special case $B=0$ the supersymmetric CP problem is solved automatically.

In fact, the special boundary conditions with vanishing superpartner masses and Aterms are theoretically well motivated. They arise, for example, in no-scale supergravity [8] or in the recently constructed higher-dimensional "gaugino mediation" models [9-11]. In gaugino mediation the MSSM matter fields are confined to a brane in higher dimensions. Supersymmetry is assumed to break on a distant parallel brane. Extra dimensional locality forbids direct couplings between the two branes and thereby suppresses all soft masses which involve MSSM matter fields (squark and slepton masses, A-terms). Gauge fields and gauginos propagate in the bulk and couple directly to supersymmetry breaking, allowing for the generation of gaugino masses. 
Extra-dimensional locality only forbids scalar masses at energies large compared to the compactification scale $M_{B C}$. At long distances the theory is four-dimensional and masses are generated from renormalization as usual. The compactification scale in gaugino mediation corresponds to a free parameter. Gauge coupling unification motivates us to choose $M_{B C}>$ $M_{G U T}$, and an upper limit on $M_{B C}$ is given by the length scale at which Nature becomes non-local, presumably the string scale or Planck scale. In no-scale supergravity the scale $M_{B C}$ at which soft scalar masses vanish is related to the string scale. In the following we will treat $M_{B C}$ as a free parameter subject to the constraint $M_{G U T} \leq M_{B C} \leq M_{\text {Planck }}$, and refer to the boundary condition of vanishing scalar masses and A terms as gaugino mediation.

Clearly this scenario is very appealing and it is crucial to ask if it is phenomenologically viable. For the particular choice of $M_{B C}=M_{G U T}$ one finds that the stau is the lightest superpartner (LSP) which is problematic because the calculated relic abundance of stable staus exceeds experimental limits by many orders of magnitude. This observation is often conceived as a failure of no-scale models and has motivated construction of models with new fields at intermediate scales to modify the renormalization group equations. In this paper we repeat the analysis for general $M_{B C} \geq M_{G U T}$ and find the good news that the problematic stau-LSP is very special to $M_{B C} \approx M_{G U T}$. For compactification scales slightly higher than $M_{G U T}$ the stau mass gets a large new contribution from running in the unified theory above $M_{G U T}$ which lifts its mass above the mass of the Bino. This results in a very satisfying cosmological picture with a Bino-LSP.

Usually, the renormalization above the GUT scale is ignored (see, however, Refs. [12-15]). There are two seemingly good reasons for this negligence, we find that both are invalid. The first reason given is that $\log \left(M_{B C} / M_{G U T}\right)$ is negligibly small compared to $\log \left(M_{G U T} / M_{\text {weak }}\right)$. However, we find that the smallness of the logarithm is compensated for by much larger group theory factors which arise in GUT theories. In particular, the right-handed sleptons receive only very small contributions from running below the GUT scale because they carry only hypercharge, but above $M_{G U T}$ they are unified into a much larger representation with large corresponding group theory factors. The second reason is that the renormalization group equations above $M_{G U T}$ are necessarily model-dependent. Obviously, GUTs have more fields than just the MSSM multiplets and the adjoint field needed to break the GUT symmetry to the Standard Model gauge group. For example, there must be additional multiplets that guarantee the splitting of the doublet and triplet Higgs fields. All such new GUT-scale fields appear with model-dependent SUSY couplings. However, this does not necessarily imply that the running of all soft parameters above the GUT scale is model dependent. As we will demonstrate in gaugino mediation most of the soft masses decouple from the unknown physics and many predictions can be made in a model-independent fashion.

In the next two sections we describe the renormalization group analysis above (Section II) and below (Section III) $M_{G U T}$ in detail. We find that the number of model-independent predictions that can be obtained is related to the form of the boundary conditions at $M_{B C}$. General gaugino mediation boundary conditions allow arbitrary soft Higgs masses [10,16], 
a B-term and gaugino masses at the scale $M_{B C}$. We show that in this case the spectrum of the first two generations and the ratios of gaugino masses can be predicted. When only $M_{1 / 2}$ and $B$ are non-zero at $M_{B C}$ one can also predict third generation scalar masses. Finally, when $B$ is also set to zero one obtains the boundary conditions of Minimal Gaugino Mediation [11]. In this case the entire superpartner spectrum can be predicted in terms of just two parameters: $M_{1 / 2}$ and $M_{B C}$. We conclude in Section IV.

\section{RENORMALIZATION ABOVE THE GUT SCALE}

In this section we discuss the renormalization of soft mass parameters above the grand unification scale, which we take to be $M_{G U T}=210^{16} \mathrm{GeV}$. We first briefly summarize the situation in a general SUSY-GUT with soft masses and then turn to gaugino dominated scenarios such as gaugino mediation or no-scale supergravity. We assume that there is a direct coupling of supersymmetry breaking to the gauginos. Then the (one-loop) "anomaly mediation" [3] contributions to superpartner masses are always negligible.

\section{A. General case}

A realistic GUT theory requires a number of new fields above the GUT scale for breaking the GUT symmetry, splitting the Higgs doublets and triplets, and possibly also for generating flavor. There exists a large number of different proposals for addressing all these problems, but unfortunately present day experiments do not allow us to single out a unique "GUT Standard Model". Not knowing the exact spectrum and couplings above the GUT scale makes it impossible to perform a reliable renormalization group calculation of all superpartner masses above the GUT scale. A conservative approach would then be to parameterize our ignorance by assuming general non-universal but GUT-symmetric superpartner masses

at $M_{G U T}$. To simplify and to avoid conflicts with experimental bounds on flavor violation one often assumes that the soft parameters are approximately flavor symmetric. This approximation can be poor for third generation scalar masses which can be significantly modified because of the large Yukawa couplings. However, the first and second generation Yukawa couplings are presumably small also above $M_{G U T}$ and can therefore be neglected in the running. Then the one-loop renormalization of the soft scalar masses for the first and second generation depends only on the unified gauge coupling and is flavor-universal. It is therefore possible to compute the running of these soft masses and one can make predictions if they are known at some high scale. The results of this running are well-known [17].

\section{B. Gaugino domination}

In gaugino-dominated scenarios the situation is different. In gaugino domination one assumes that the scalar masses as well as the tri-linear soft terms vanish at some high scale 
$M_{B C}$, and their low-energy values are generated from the renormalization group. Thus at the scale $M_{B C} \geq M_{G U T}$ the non-vanishing mass parameters are the universal gaugino mass $M_{1 / 2}$ and the supersymmetry preserving and violating Higgs mass parameters $\mu$ and $B$. In addition, one could also have soft masses for the Higgs fields, but for the time being let us assume that $m_{H_{u}}$ and $m_{H_{d}}$ are zero at $M_{B C}$.

As we will see below, $\mu$ does not enter any renormalization group equation for the soft terms neither above nor below $M_{G U T}$. Since we do not have a physical principle which tells us the value of $\mu$ at the high scale, this means that there is no need to run the value of $\mu$. In the phenomenological analysis we simply work with its low-energy value. $B$ also does not enter any renormalization group equations. However, Minimal Gaugino Mediation predicts $B=0$ at the high scale, it is therefore important to predict the low-energy value of $B$ from the renormalization group running between $M_{B C}$ to $M_{\text {weak }}$.

The evolution of the unified gaugino mass between $M_{B C}$ and $M_{G U T}$ is easily determined because at one loop $M_{1 / 2}$ simply tracks the evolution of the unified gauge coupling

$$
\frac{d}{d t} \frac{1}{g^{2}}=-2 b_{G U T} \quad \frac{d}{d t} \frac{M_{1 / 2}}{g^{2}}=0 .
$$

Here we defined $t=1 /\left(16 \pi^{2}\right) \log \left(M / M_{G U T}\right)$. The renormalization group equations for all other soft masses above $M_{G U T}$ are $^{1}$

$$
\begin{aligned}
\frac{d}{d t} m_{10}^{2} & =\frac{3}{2} \frac{d}{d t} m_{5}^{2}=\frac{3}{2} \frac{d}{d t} m_{5}^{2}=-\frac{144}{5} g^{2} M_{1 / 2}^{2}, \\
\frac{d}{d t} A_{u} & =\frac{8}{7} \frac{d}{d t} A_{d}=2 \frac{d}{d t} B=\frac{192}{5} g^{2} M_{1 / 2},
\end{aligned}
$$

for the case of $S U(5)$ and

$$
\begin{aligned}
\frac{d}{d t} m_{16}^{2} & =\frac{5}{4} \frac{d}{d t} m_{10}^{2}=-45 g^{2} M_{1 / 2}^{2}, \\
\frac{d}{d t} A_{u, d} & =\frac{7}{4} \frac{d}{d t} B=63 g^{2} M_{1 / 2},
\end{aligned}
$$

for $S O(10)$. On the right-hand side of these equations we assumed that all soft masses except $M_{1 / 2}$ are negligible. This is a very good approximation at energies near $M_{B C}$ where they all vanish but becomes worse if significant scalar masses are generated from the renormalization group running.

Note that all dependence on unknown couplings above the GUT scale has dropped out of Eqs. (2.3) and (2.5). The remaining model dependence lies in the choice of grand unified group and in the evolution of the GUT gauge coupling (specifically $b_{G U T}$ ).

\footnotetext{
${ }^{1}$ We define the soft mass parameters $A$ and $B$ such that they multiply the Yukawa couplings and $\mu$ in the Lagrangian, respectively. For example, $\mathcal{L}_{\text {soft }} \sim A_{t} Y_{t} \mathbf{1 0 1 0} \mathbf{5}_{H_{U}}+B \mu \mathbf{5}_{H_{U}} \overline{\mathbf{5}}_{H_{D}}$.
} 
The solutions to these renormalization group equations are most easily written by using Eq. (2.1) to replace $M_{1 / 2}(\mu) \rightarrow M_{1 / 2}\left(M_{G U T}\right) \alpha(\mu) / \alpha_{G U T}$ and defining

$$
\begin{aligned}
& I_{4}=\int_{0}^{t_{B C}} g^{4} d t=\alpha_{G U T}^{2} \log \left(\frac{M_{B C}}{M_{G U T}}\right) \frac{1}{1-\frac{b_{G U T} \alpha_{G U T}}{2 \pi} \log \left(\frac{M_{B C}}{M_{G U T}}\right)}, \\
& I_{6}=\int_{0}^{t_{B C}} g^{6} d t=4 \pi \alpha_{G U T}^{3} \log \left(\frac{M_{B C}}{M_{G U T}}\right) \frac{1-\frac{b_{G U T} \alpha_{G U T}}{4 \pi} \log \left(\frac{M_{B C}}{M_{G U T}}\right)}{\left(1-\frac{b_{G U T} \alpha_{G U T}}{2 \pi} \log \left(\frac{M_{B C}}{M_{G U T}}\right)\right)^{2}} .
\end{aligned}
$$

We find for $S U(5)$

$$
\begin{aligned}
m_{10}^{2} & =\frac{3}{2} m_{5}^{2}=\frac{3}{2} m_{5}^{2}=\frac{144}{5} M_{1 / 2}^{2} I_{6} \\
A_{u} & =\frac{8}{7} A_{d}=2 B=-\frac{192}{5} M_{1 / 2} I_{4}
\end{aligned}
$$

and for $S O(10)$

$$
\begin{aligned}
& m_{16}^{2}=\frac{5}{4} m_{10}^{2}=45 M_{1 / 2}^{2} I_{6}, \\
& A_{u, d}=\frac{7}{4} B=-63 M_{1 / 2} I_{4},
\end{aligned}
$$

where from now on $M_{1 / 2}$ stands for the unified gaugino mass evaluated at $M_{G U T}$.

Now all soft supersymmetry breaking at the GUT scale is determined in terms of the five parameters $M_{1 / 2}, \mu, B, I_{4}$ and $I_{6} . I_{4}$ and $I_{6}$ are given in terms of the two parameters $b_{G U T}$ and $\log \left(M_{B C} / M_{G U T}\right)$. Note that $I_{4}$ and $I_{6}$ are small enough for our approximation (ignoring all soft masses except for the gaugino mass) to be valid unless the denominators in Eqs.( 2.6) and (2.7) go to zero. But the same vanishing denominators also appear in the evolution of the gauge coupling. Therefore our approximation is good if and only if the theory stays perturbative up to the mass scale $M_{B C}$. For example, for $\frac{M_{B C}}{M_{G U T}}=10$ perturbativity allows a beta function coefficient $b_{G U T}$ as large as 50. Note that this leaves sufficient room for nonminimal Higgs sectors above $M_{G U T}$ because the minimal $S U(5)$ theory only has $b_{G U T}=-3$, an extra generation adds 2 and extra adjoint superfields contribute 5. If the theory above the GUT scale has large new Yukawa couplings, for example a coupling of $H_{u}$ and $H_{d}$ to a GUT adjoint superfield, then terms proportional to scalar masses on the right hand side can make contributions to scalar masses which become important for large $M_{B C} \sim M_{\text {Planck }}$. This can be seen from the numerical solutions to the renormalization group equations of the minimal $S U(5)$ model with gaugino mediation boundary condition presented in Ref. [15].

This discussion changes if we allow non-zero supersymmetry violating masses $m_{H_{u}}^{2}$ and $m_{H_{d}}^{2}$ at $M_{B C}$. Not only is the running of these masses very sensitive to new physics above the GUT scale (such as the doublet-triplet splitting mechanism or a non-minimal GUT Higgs sector), but also the third generation scalar masses are now model dependent because of contributions proportional to the large Yukawa couplings. Thus, in this case modelindependent predictions are only possible for the first and second generation scalar masses. 


\section{THE SPECTRUM}

In this section we discuss the superpartner masses which result from running from the compactification scale all the way down to the weak scale. Throughout the discussion we assume that the gravitino mass is larger than the gaugino masses. This assumption is important for the phenomenology of the model. Parametrically, in gaugino mediation one finds $m_{3 / 2}=\sqrt{V} m_{1 / 2}$ where $V>8$ is the volume of the extra dimensions in fundamental Planck units $[9,10]$. We proceed by presenting three qualitatively different scenarios. All three scenarios have vanishing squark and slepton masses and A-terms at the high scale but they differ in the assumptions made about the Higgs sector. In scenario $\mathbf{A}$ we allow general soft Higgs mass parameters $m_{H_{u}}^{2}, m_{H_{d}}^{2}, B$, and $\mu$. In $\mathbf{B}$ we specialize to models with vanishing non-holomorphic masses $m_{H_{u}}^{2}=m_{H_{d}}^{2}=0$. And in $\mathbf{C}$ we also assume $B=0$, so that the only remaining mass parameters of the model are $M_{1 / 2}, \mu, M_{B C}$ and $M_{G U T}$. In the last section we also give three example superpartner spectra corresponding to representative sets of model input parameters which satisfy the Mg̃M boundary conditions.

We solve the one-loop renormalization group equations of the MSSM [18] below the GUT scale numerically. We include the effects of third generation Yukawa couplings and neglect the smaller Yukawa couplings. The GUT scale boundary conditions for all couplings follow from the analysis in Section II. Note that there are no threshold contributions to the supersymmetry breaking parameters in the $\overline{D R}$ renormalization scheme from integrating out the heavy GUT gauge bosons and gauginos. Explicitly, this follows because diagrams renormalizing the scalar masses with heavy GUT gauginos in a loop have vanishing finite pieces in $\overline{D R}$. At the weak scale we use a one-loop improvement for the Higgs potential $[19,20]$ which captures the effect of top loops below the stop mass threshold. The top loops modify the coefficient of the $\left(H_{u}^{\dagger} H_{u}\right)^{2}$ quartic term and represent the dominant correction to the mass of the lightest Higgs particle. The accuracy of this approximation is to better than 10 $\mathrm{GeV}[21]$, when the running top quark mass $m_{t o p}\left(m_{t o p}\right)$ is used for the calculation.

\section{A. General Higgs mass parameters}

When we allow Higgs masses $m_{H_{u}}^{2}$ and $m_{H_{d}}^{2}$ at the scale $M_{B C}$ then only the soft masses of the first two generations and gaugino masses can be predicted without knowledge of details of the GUT physics. It is convenient to use the average soft Higgs mass $\left(m_{H_{u}}^{2}+m_{H_{d}}^{2}\right) / 2$ and the difference $m_{H_{u}}^{2}-m_{H_{d}}^{2}$ as input parameters. The average Higgs mass does not contribute directly to first and second generation scalar masses. Indirectly, it does contribute to scalar masses through weak scale D-terms, but for large enough tan $\beta$ these D-terms can be written universally in terms of the $\mathrm{W}$ and $\mathrm{Z}$ masses. The difference does contribute because it generates a D-term for hypercharge. This D-term is proportional to the renormalization group invariant quantity $S$ defined as

$$
S=m_{H_{u}}^{2}-m_{H_{d}}^{2}+\operatorname{Tr}\left(m_{Q}^{2}-2 m_{U}^{2}+m_{E}^{2}+m_{D}^{2}-m_{L}^{2}\right)=m_{H_{u}}^{2}-\left.m_{H_{d}}^{2}\right|_{M_{G U T}} .
$$


where the second equality is valid only at the GUT scale because the squark and slepton masses in the trace are GUT symmetric and therefore drop out of the equation. The D-term mass shift for each scalar at the weak scale is simply

$$
\delta m_{i}^{2}=-\frac{6}{5} y_{i} S \int_{t_{\text {weak }}}^{0} g_{1}^{2} d t \simeq-.078 y_{i} S .
$$

We first specialize to the case with no D-term for hypercharge, i.e. $m_{H_{u}}^{2}=m_{H_{d}}^{2}$ at $M_{G U T}$. Figure 1 illustrates the evolution of soft masses for the first two generations and the gauginos from $M_{B C}>M_{G U T}$ to the weak scale. At $M_{B C}$ the soft masses vanish and evolve according to $S U(5)$ RGEs down to $M_{G U T}$. For the purpose of this plot we assumed $S U(5)$ unification therefore the $\overline{\mathbf{5}}$ and $\mathbf{1 0}$ evolve at different rates. The gaugino masses are unified between $M_{B C}$ and $M_{G U T}$. Below $M_{G U T}$ the RGEs respect only the symmetries of the Standard Model. The evolution depends on the gauge charges of the fields. Gaugino masses and scalar masses are proportional to the squares of the gauge couplings. As a result, colored fields are always heaviest and have masses about four times larger than fields with only hypercharge.

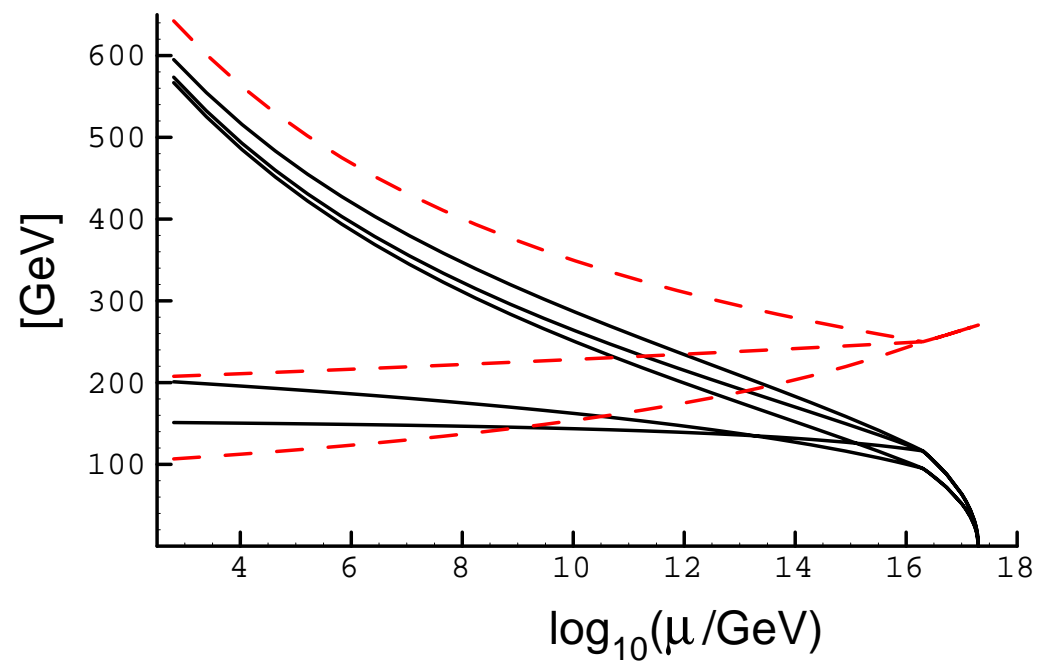

FIG. 1. Evolution of the soft masses of the first two generations (solid) and the gauginos (dashed) as a function of renormalization scale $\mu$. The input parameters are $M_{1 / 2}=250 \mathrm{GeV}$, $M_{B C}=2 \cdot 10^{17} \mathrm{GeV}$, and vanishing hypercharge D-term $S=0$. The scalar fields are, from the lightest to heaviest at the weak scale, the right-handed selectron, the left-handed sleptons, the right-handed down and up squarks, and the left-handed squarks. The gaugino masses start at a nonzero value at $M_{B C}$. At the weak scale the gluino is heaviest and the Bino lightest.

The effects of the running above the GUT scale are depicted in Figure 2. Scalar masses receive additive GUT-symmetric contributions from the running above $M_{G U T}$. This effect is most important for scalars which do not receive large masses from running below the GUT scale. The mass shifts are proportional to the Casimirs of the corresponding unified representations, thus they are larger in $S O(10)$ compared to $S U(5)$. 


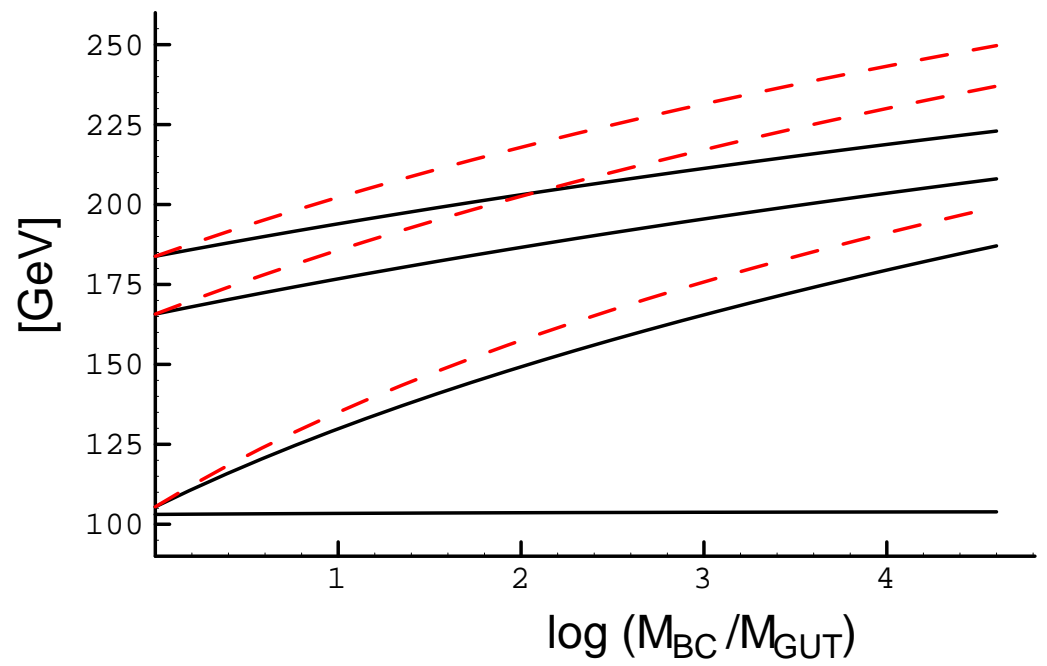

FIG. 2. Weak scale superpartner masses as a function of $\log \left(M_{B C} / M_{G U T}\right)$. The common gaugino mass is $250 \mathrm{GeV}$ at $M_{G U T}$ and we take $S=0$. The lightest particle is the neutralino, its mass is independent of $M_{B C}$. The sleptons are, from the lightest to the heaviest, the right-handed selectron, the left-handed sneutrino, and the left-handed selectron. The solid lines correspond to the running in $S U(5)$, dashed lines correspond to $S O(10)$.

A non-vanishing D-term introduces the additional input parameter $S$. The $S$-dependence of the first and second generation scalar masses is easily accounted for by using equation (3.2). The effects of the D-term are largest for the lightest superpartners. In Figure 3 we show the slepton masses for the case of $S U(5)$ GUT group and three different values of $S$. The mass shifts from the hypercharge D-term are in opposite directions for left- and right-handed sleptons.

Note that the hypercharge D-terms also contribute to stau masses and may be responsible for lifting the right-handed stau mass above the Bino mass in models where $M_{B C}=M_{G U T}$. This has been used in Refs. $[10,16]$.

$$
\text { B. } m_{H_{u}}^{2}=m_{H_{d}}^{2}=0
$$

When the Higgs masses are zero at $M_{B C}$, the Yukawa couplings do not significantly contribute to the running above the GUT scale. Therefore the running for all three generations can be computed model independently. For fixed $M_{1 / 2}$ and $M_{B C}, B$ and $\tan \beta$ are related at the minimum of the Higgs potential. Thus, we can express $B$ in terms of $\tan \beta$ or vice versa.

The relation between $B$ at the high scale and $\tan \beta$ is depicted in Figure 4 for different values of $M_{B C}$. It is clear that a value of $B$ can be picked for any values of $\tan \beta$ and $M_{B C}$. It is therefore more convenient to treat $\tan \beta$ as an input parameter, as is usually done in analyzing supersymmetric theories.

Since the ratio of the bottom and top Yukawa couplings depends on $\tan \beta$ the masses of the third generation particles vary with $\tan \beta$. In particular, the mixing between left- 


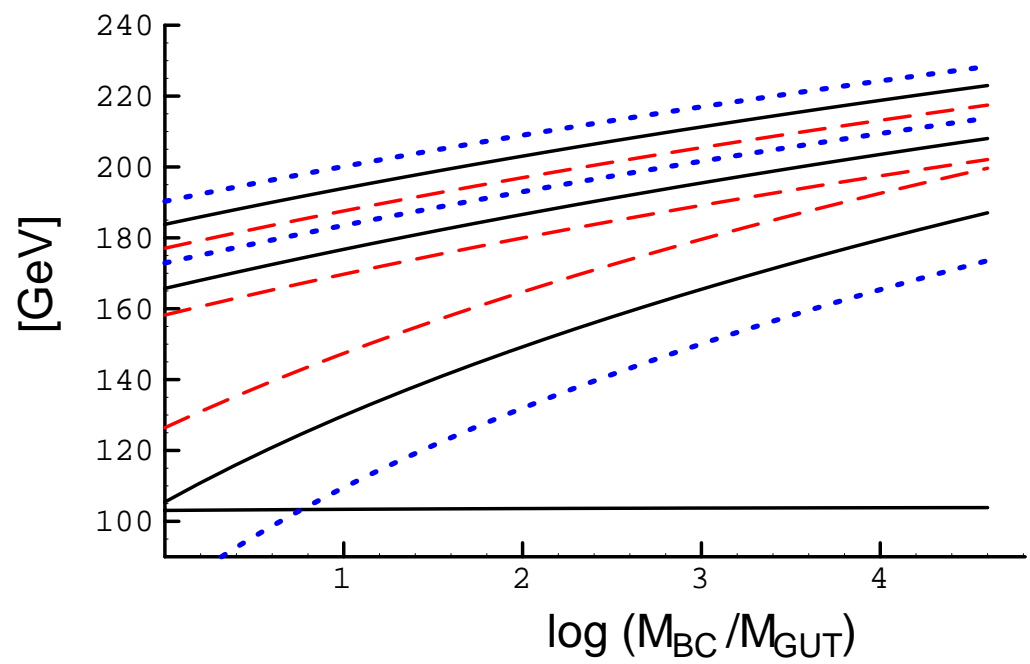

FIG. 3. Weak scale superpartner masses as a function of $\log \left(M_{B C} / M_{G U T}\right)$ for three different values of $S=-M_{1 / 2}^{2}, 0,+M_{1 / 2}^{2}$. We take $M_{1 / 2}=250 \mathrm{GeV}$ and GUT group $S U(5)$. The lightest particle is the neutralino, its mass is independent of $M_{B C}$. The other solid lines correspond to, from lightest to heaviest, the right-handed selectron, the left-handed sneutrino, and the left-handed selectron for $S=0$, as in Figure 2. Dashed lines correspond to $S=-M_{1 / 2}^{2}$ and dotted lines to $S=+M_{1 / 2}^{2}$. Note that left- and right-handed slepton masses are shifted in opposite directions.

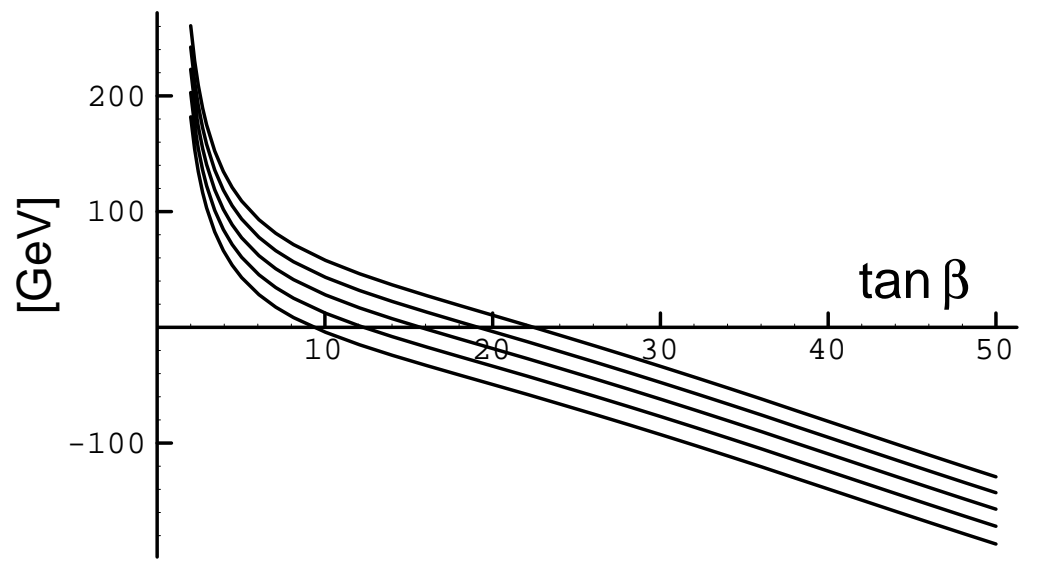

FIG. 4. The dependence of $B$, evaluated at $M_{B C}$, on $\tan \beta$. We take $M_{1 / 2}=250 \mathrm{GeV}$, the different curves correspond to $\log \left(M_{B C} / M_{G U T}\right)=0,1,2,3,4$ from left to right, respectively.

and right-handed sleptons increases with $\tan \beta$. As a result one of the mass eigenstates becomes lighter with increasing $\tan \beta$. Figure 5 illustrates this strong dependence in case of the right-handed stau.

$$
\text { C. } m_{H_{u}}^{2}=m_{H_{d}}^{2}=0, B=0
$$

In minimal gaugino mediation all soft terms except for $M_{1 / 2}$ (and $\mu$ ) vanish at $M_{B C}$. The values of $\tan \beta$ which correspond to $B=0$ can be determined easily from Figure 4 


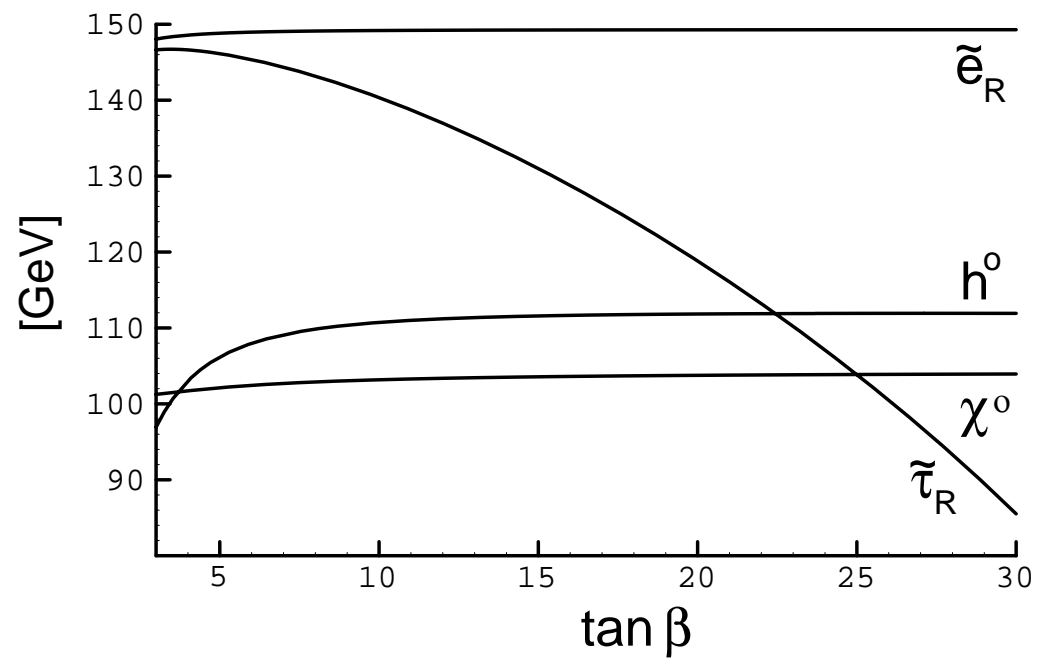

FIG. 5. Masses of the right-handed selectron, stau, the lightest Higgs and neutralino as a function of tan beta. The common gaugino mass is $250 \mathrm{GeV}$ at $M_{G U T}$ and $\log \left(M_{B C} / M_{G U T}\right)=2$.

as a function of $M_{B C}$. The two remaining parameters are $M_{1 / 2}$ and the scale $M_{B C}$. With only two free parameters the theory is highly predictive. To a good approximation $M_{1 / 2}$ sets the linear scale of all superpartner masses. ${ }^{2}$ The second parameter, $\log \left(M_{B C} / M_{G U T}\right)$, increases the slepton masses relative to gaugino masses. A more detailed discussion of Mg̃M is contained in Refs. [11,15].

For demonstration, we present the spectra for three sample points of the parameter space in Table I. The first point with $M_{1 / 2}=200 \mathrm{GeV}$ ("light M g̃M" scenario) is in the lower range of experimentally allowed values of $M_{1 / 2}$. This point will be probed in the near future by the ongoing Higgs search at LEP II. The lighter chargino and the second lightest neutralino can be observed at the Tevatron in the $p p \rightarrow \chi_{1}^{ \pm} \chi_{2}^{0} \rightarrow 3 l$ channel. While the chargino and neutralino in the "light" scenario might evade Run II they are certainly within the reach of Run III [22]. Our second and third points, with $M_{1 / 2}=300,500 \mathrm{GeV}$ - "intermediate

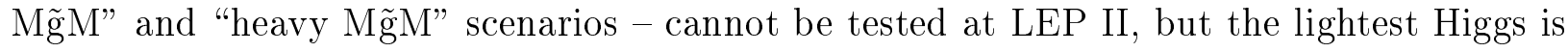
within the reach of Run II at the Tevatron. The superpartners corresponding to the second and third study points are too heavy to be seen at LEP or the Tevatron, but they are easily within the reach of both LHC and NLC.

One can set an upper bound on $M_{1 / 2} \lesssim 600 \mathrm{GeV}$ by requiring that the relic abundance of Mg̃M Bino-LSPs does not contribute more than 0.1 to 0.3 to critical density [11]. We find that in our "light" scenario Binos are not abundant enough to account for all cold dark matter, whereas the "intermediate" and "heavy" scenarios are in the preferred region.

\footnotetext{
${ }^{2}$ Note that the lightest Higgs mass does not scale linearly with $M_{1 / 2}$, because at tree level the Higgs mass is bounded by $M_{Z}$. Radiative corrections to the Higgs potential from top and stop loops introduce a logarithmic dependence on $M_{1 / 2}$.
} 


\begin{tabular}{|c|c|c|c|c|c|c|c|c|c|c|c|}
\hline \multicolumn{4}{|c|}{ I. "light Mg̃M" } & \multicolumn{4}{|c|}{ II. "intermediate Mg̃M" } & \multicolumn{4}{|c|}{ III. "heavy Mg̃M" } \\
\hline$\overline{\text { Field }}$ & mass & Field & $\overline{\text { mass }}$ & Field & mass & Field & mass & Field & mass & Field & $\overline{\operatorname{mass}}$ \\
\hline$\widetilde{g}$ & 520 & & & $\widetilde{g}$ & 780 & & & $\widetilde{g}$ & 1300 & & \\
\hline$\tilde{\chi}_{1}^{ \pm}$ & 147 & $\tilde{\chi}_{2}^{ \pm}$ & 317 & $\tilde{\chi}_{1}^{ \pm}$ & 235 & $\tilde{\chi}_{2}^{ \pm}$ & 457 & $\tilde{\chi}_{1}^{ \pm}$ & 406 & $\tilde{\chi}_{2}^{ \pm}$ & 750 \\
\hline$\widetilde{\chi}_{1}^{0}$ & 82 & $\widetilde{\chi}_{2}^{0}$ & 148 & $\widetilde{\chi}_{1}^{0}$ & 125 & $\tilde{\chi}_{2}^{0}$ & 236 & $\tilde{\chi}_{1}^{0}$ & 211 & $\widetilde{\chi}_{2}^{0}$ & 406 \\
\hline$\widetilde{\chi}_{3}^{0}$ & 294 & $\widetilde{\chi}_{4}^{0}$ & 315 & $\widetilde{\chi}_{3}^{0}$ & 441 & $\widetilde{\chi}_{4}^{0}$ & 456 & $\tilde{\chi}_{3}^{0}$ & 739 & $\widetilde{\chi}_{4}^{0}$ & 750 \\
\hline$\widetilde{u}_{L}$ & 478 & $\widetilde{u}_{R}$ & 463 & $\widetilde{u}_{L}$ & 720 & $\widetilde{u}_{R}$ & 696 & $\widetilde{u}_{L}$ & 1188 & $\widetilde{u}_{R}$ & 1145 \\
\hline$\widetilde{d}_{L}$ & 485 & $\widetilde{d}_{R}$ & 460 & $\widetilde{d}_{L}$ & 725 & $\widetilde{d}_{R}$ & 689 & $\widetilde{d}_{L}$ & 1191 & $\widetilde{d}_{R}$ & 1138 \\
\hline$\tilde{t}_{1}$ & 351 & $\tilde{t}_{2}$ & 501 & $\tilde{t}_{1}$ & 535 & $\tilde{t}_{2}$ & 703 & $\tilde{t}_{1}$ & 906 & $\tilde{t}_{2}$ & 1117 \\
\hline$\widetilde{b}_{1}$ & 437 & $\widetilde{b}_{2}$ & 497 & $\widetilde{b}_{1}$ & 664 & $\widetilde{b}_{2}$ & 735 & $\widetilde{b}_{1}$ & 1121 & $\widetilde{b}_{2}$ & 1195 \\
\hline$\widetilde{e}_{L}$ & 167 & $\widetilde{e}_{R}$ & 126 & $\widetilde{e}_{L}$ & 245 & $\widetilde{e}_{R}$ & 183 & $\widetilde{e}_{L}$ & 373 & $\widetilde{e}_{R}$ & 235 \\
\hline$\widetilde{\nu}_{e}$ & 147 & $\widetilde{\nu}_{\tau}$ & 147 & $\widetilde{\nu}_{e}$ & 231 & $\widetilde{\nu}_{\tau}$ & 231 & $\widetilde{\nu}_{e}$ & 364 & $\widetilde{\nu}_{\tau}$ & 364 \\
\hline$\widetilde{\tau}_{1}$ & 104 & $\widetilde{\tau}_{2}$ & 178 & $\widetilde{\tau}_{1}$ & 162 & $\widetilde{\tau}_{2}$ & 253 & $\widetilde{\tau}_{1}$ & 224 & $\widetilde{\tau}_{2}$ & 375 \\
\hline$h^{0}$ & 108 & $H^{0}$ & 294 & $h^{0}$ & 115 & $H^{0}$ & 453 & $h^{0}$ & 122 & $H^{0}$ & 793 \\
\hline$A^{0}$ & 294 & $H^{ \pm}$ & 312 & $A^{0}$ & 453 & $H^{ \pm}$ & 464 & $A^{0}$ & 793 & $H^{ \pm}$ & 800 \\
\hline
\end{tabular}

TABLE I. Masses of superpartners, in GeV, for Mg̃M study points I, II and III. They correspond to parameter values "light" $\left(M_{1 / 2}=200 \mathrm{GeV}, M_{B C} / M_{G U T}=10, \tan \beta=17\right)$, "intermediate" $\left(M_{1 / 2}=300 \mathrm{GeV}, M_{B C} / M_{G U T}=10, \tan \beta=17\right)$, and "heavy" $\left(M_{1 / 2}=500 \mathrm{GeV}\right.$, $\left.M_{B C} / M_{G U T}=2, \tan \beta=12\right)$, respectively.

\section{CONCLUSIONS}

We conclude that the MSSM with gaugino mediated supersymmetry breaking is not only phenomenologically viable, but it also has a number of very attractive features:

- The model solves the supersymmetric flavor problem because the squark and slepton masses which are generated from the renormalization group evolution are sufficiently degenerate (and aligned [23]).

- It is theoretically well motivated. The vanishing of the scalar masses and the $A$ terms at high scales is a natural prediction of models with extra dimensions where MSSM gauge fields are bulk fields, whereas the MSSM matter fields and the supersymmetry breaking mechanism are localized on separate branes.

- The Bino-LSP of these models makes a good cold dark matter candidate. Cosmic abundances in the range $\Omega h^{2}=.1-.3$ are obtained for right-handed selectron masses in the range $150-250 \mathrm{GeV}$ as shown in [11,24].

- The model is very predictive because superpartner masses depend only on a small number of input parameters. For example, in Mõg all superpartner masses can be 
computed in terms of two parameters $M_{1 / 2}$ and $\log \left(M_{B C} / M_{G U T}\right)$. In the more general case with non-vanishing soft Higgs masses at $M_{B C}$ all gaugino masses and the first and second generation scalar masses are predicted in terms of the same two parameters and possibly a hypercharge D-term $S$.

In our analysis of the model the renormalization group running above the GUT scale was essential for determining the masses of the lightest scalar superpartners. Its most important effect is that it raises the stau mass above the mass of the Bino. Precise measurements of the superpartner masses would allow a determination of the small contributions from running above the GUT scale [25]. Consistency with the gaugino mediation predictions would constitute a decisive test of the scenario and allow an indirect measurement of the GUT gauge group.

The framework is predictive because at the one-loop level above-the-GUT-scale model dependence decouples from the soft supersymmetry breaking terms. This is a consequence of vanishing scalar masses at $M_{B C}$ and would not be true for more general SUSY GUTs. If the soft Higgs mass parameters $m_{H_{u}}^{2}$ and $m_{H_{d}}^{2}$ are non-vanishing at the GUT scale then the third generation scalar masses and Higgs masses become model-dependent, but first and second generation scalar masses as well as gaugino masses can still be predicted. In this

case the hypercharge D-term proportional to $m_{H_{u}}^{2}-m_{H_{d}}^{2}$ also plays an important role in determining the lightest superpartner masses.

We have left studies of the collider phenomenology of these models for future work. In particular, it would be interesting to determine the most promising signatures which allow tests of this scenario and the implications for future colliders. As part of this analysis a more accurate treatment of the renormalization group (two-loops) and weak scale threshold effects would allow sharpened predictions for superpartner masses. This more accurate analysis is necessary for comparison of our Higgs mass prediction with LEP II bounds. We also expect interesting predictions for (and constraints from) flavor violating transitions such as $b \rightarrow s \gamma$ and $\mu \rightarrow e \gamma$.

\section{ACKNOWLEDGMENTS}

We thank Howard Baer, Markus Luty, Elazzar Kaplan, Konstantin Matchev, Michael Peskin, Raman Sundrum, and Jim Wells for useful discussions. MS is supported by the DOE under contract DE-AC03-76SF00515. WS is supported by the DOE under contract DOE-FG03-97ER40506. 


\section{REFERENCES}

[1] M. Dine, W. Fischler, and M. Srednicki, Nucl. Phys. B189, 575 (1981); M. Dine and M. Srednicki, Nucl. Phys. B202, 238 (1982).

[2] M. Dine and A. Nelson, Phys. Rev. D48, 1277 (1993), hep-ph/9303230; M. Dine, A. E. Nelson, and Y. Shirman, Phys. Rev. D51, 1362 (1995), hep-ph/9408384; M. Dine, A. E. Nelson, Y. Nir, and Y. Shirman, Phys. Rev. D53, 2658 (1996), hep-ph/9507378.

[3] L. Randall and R. Sundrum, hep-th/9810155; G. Giudice, M. Luty, H. Murayama, and R. Rattazzi, JHEP 9812:027 (1998), hep-ph/9810442;

[4] G. Dvali and A. Pomarol, Phys. Rev. Lett. 77, 3728 (1996), hep-ph/9607383; P. Binetruy and E. Dudas, Phys. Lett. B389, 503 (1996),hep-th/9607172; A. G. Cohen, D. B. Kaplan, and A. E. Nelson, Phys. Lett. B388, 588 (1996), hep-ph/9607394.

[5] L. Hall, J. Lykken, and S. Weinberg, Phys. Rev. D27, 2359 (1983).

[6] M. Dine, A. Kagan, and S. Samuel, Phys. Lett. B243, 250 (1990).

[7] L. Hall and L. Randall, Phys. Rev. Lett. 65, 2939 (1990).

[8] J. Ellis, C. Kounnas, and D.V. Nanopoulos, Nucl. Phys. B247, 373 (1984);

A.B. Lahanas and D.V. Nanopoulos,Phys. Rept. 145, 1 (1987).

[9] D.E. Kaplan, G.D. Kribs, and M. Schmaltz, hep-ph/9911293.

[10] Z. Chacko, M. Luty, A.E. Nelson, and E. Pontón, JHEP 0001: 003 (2000), hep-ph/9911323.

[11] M. Schmaltz and W. Skiba, hep-ph/0001172.

[12] N. Polonsky and A. Pomarol, Phys. Rev. Lett. 73, 2292 (1994), hep-ph/9406224; Phys. Rev. D51, 6532 (1995), hep-ph/9410231.

[13] Y. Kawamura, H. Murayama, and M. Yamaguchi, Phys. Rev. D51, 1337 (1995), hep$\mathrm{ph} / 9406245$.

[14] R. Barbieri and L. Hall, Phys. Lett. B338, 212 (1994), hep-ph/9408406;

R. Barbieri, L. Hall, and A. Strumia, Nucl. Phys. B445, 219 (1995), hep-ph/9501334.

[15] H. Baer, M. Diaz, P. Quintana, and X. Tata, JHEP 0004, 016 (2000), hep-ph/0002245.

[16] D. E. Kaplan and T. M. Tait, hep-ph/0004200.

[17] S. Martin, hep-ph/9709356.

[18] V. Barger, M. Berger, and P. Ohmann, Phys. Rev. D49, 4908 (1994), hep-ph/9311269. S. Martin and M. Vaughn, Phys. Rev. D50, 2282 (1994), hep-ph/9311340. 
[19] H. Haber and R. Hempfling, Phys. Rev. D48, 4280 (1993), hep-ph/9307201.

[20] D. Pierce, J. Bagger, K. Matchev, and R. Zhang, Nucl. Phys. B491, 3 (1997), hep$\mathrm{ph} / 9606211$.

[21] H. Haber, R. Hempfling, and A. Hoang, Z. Phys. C75, 539 (1997), hep-ph/9609331.

[22] S. Abel et al., SUGRA Working Group Collaboration, hep-ph/0003154; V. Barger and C. Kao, Phys. Rev. D60, 115015 (1999), hep-ph/9811489; K. Matchev and D. Pierce, Phys. Rev. D60, 075004 (1999), hep-ph/9904282; Phys. Lett. B467, 225 (1999), hep$\mathrm{ph} / 9907505$.

[23] Y. Nir and N. Seiberg, Phys. Lett. B309, 337 (1993), hep-ph/9304307.

[24] J.D. Wells, Phys. Lett. B443, 196 (1998), hep-ph/9809504; M. Drees and M. Nojiri, Phys. Rev. D47, 376 (1993), hep-ph/9207234.

[25] J.D. Wells, talk at Berkeley 2000, March 29 - 31, 2000, Berkeley, California, USA; S.P. Martin and J.D. Wells, to appear. 